CFTP/13-013

UWThPh-2013-14

\title{
A new $A_{4}$ model for lepton mixing
}

\author{
P.M. Ferreira, ${ }^{(1,2) *}$ L. Lavoura, ${ }^{(3) \dagger}$ and P.O. Ludl ${ }^{(4) \ddagger}$
}

(1) Instituto Superior de Engenharia de Lisboa 1959-007 Lisbon, Portugal

(2) Centre for Theoretical and Computational Physics, University of Lisbon 1649-003 Lisbon, Portugal

${ }^{(3)}$ Universidade de Lisboa, Instituto Superior Técnico, CFTP 1049-001 Lisbon, Portugal

(4) University of Vienna, Faculty of Physics, Boltzmanngasse 5, A-1090 Vienna, Austria

\section{October 2013}

\begin{abstract}
We present a new model of the lepton sector that uses a family symmetry $A_{4}$ to make predictions for lepton mixing which are invariant under any permutation of the three flavours. We show that those predictions broadly agree with the experimental data, leading to a largish $\sin ^{2} \theta_{12} \gtrsim 0.34$, to $|\cos \delta| \gtrsim 0.7$, and to $\left|0.5-\sin ^{2} \theta_{23}\right| \gtrsim 0.08$; $\cos \delta$ and $0.5-\sin ^{2} \theta_{23}$ are predicted to have identical signs.
\end{abstract}

\footnotetext{
*E-mail: ferreira@cii.fc.ul.pt

${ }^{\dagger}$ E-mail: balio@cftp.ist.utl.pt

${ }^{\ddagger}$ E-mail: patrick.ludl@univie.ac.at
} 
The experimental discovery that the lepton mixing angle $\theta_{13}$ is nonzero [1] caused a profound change in the subject of flavour models for the lepton mass matrices. Many older models ceased to be valid. New models had to be built; some recent examples utilizing the horizontal symmetry group $A_{4}$ are collected in refs. [2, 3]. Many of those models use 'flavons' and nonrenormalizable Lagrangians [2]; in most remaining models [3] there are Higgs doublets at the Fermi scale placed in triplets of the horizontal symmetry 1 In this paper we present a model that contains only renormalizable terms and only Higgs doublets which are singlets of $A_{4}$.

For any $n \times n$ non-singular matrix $M=\left[M_{\alpha \beta}\right]$, one may define a matrix $A=\left[A_{\alpha \beta}\right]$ through

$$
A_{\alpha \beta}=M_{\alpha \beta}\left(M^{-1}\right)_{\beta \alpha},
$$

where no sum over either $\alpha$ or $\beta$ is implied. It is obvious from its definition that $A$ satisfies

$$
\sum_{\alpha=1}^{n} A_{\alpha \beta}=\sum_{\beta=1}^{n} A_{\alpha \beta}=1 .
$$

The matrix $A$ is invariant under

$$
M \rightarrow X M Y,
$$

where $X$ and $Y$ are diagonal non-singular matrices.

For our purposes, $M$ is the (effective) Majorana mass matrix of the three light neutrinos in the weak basis where the charged-lepton mass matrix is diagonal. Therefore, $n=3$, the indices $\alpha$ and $\beta$ are in the range $\{e, \mu, \tau\}, M$ and $A$ are symmetric, and $X=Y$ in the transformation (3) 2.2 The model in this paper predicts 3

$$
A_{e \mu}=A_{e \tau}=A_{\mu \tau},
$$

hence

$$
A=\left(\begin{array}{ccc}
1-2 t & t & t \\
t & 1-2 t & t \\
t & t & 1-2 t
\end{array}\right)
$$

\footnotetext{
${ }^{1}$ An exception is the Babu-Ma-Valle model [4], in which the Higgs doublets are $A_{4}$ invariant. That model depends on renormalization to produce realistic neutrino masses and mixings.

${ }^{2}$ The matrix $A$ was also used in this context in ref. [5].

${ }^{3}$ Equations (5) may alternatively be stated as

$$
M_{e e}\left(M_{\mu \tau}\right)^{2}=M_{\mu \mu}\left(M_{e \tau}\right)^{2}=M_{\tau \tau}\left(M_{e \mu}\right)^{2} .
$$


where $t$ is in general a complex number. Moreover, through the imposition of an additional $C P$ symmetry on our model, $t$ may be made to be real. We shall show that the conditions (5) fit the experimental data rather well 4

Our model has the usual Standard-Model leptonic multiplets $\alpha_{R}:(\mathbf{1},-1)$ and $D_{\alpha L}:(\mathbf{2},-1 / 2) 5$ It has, besides, three right-handed neutrinos $\nu_{\alpha R}$ : $(\mathbf{1}, 0)$. The scalar sector is composed of three Higgs doublets $\phi_{k}:(\mathbf{2}, 1 / 2)$, where $k \in\{1,2,3\}$. Their conjugate doublets are $\tilde{\phi}_{k} \equiv i \tau_{2} \phi_{k}^{*}:(\mathbf{2},-1 / 2)$. In our model there are, besides, three real scalars $\sigma_{\alpha}:(\mathbf{1}, 0)$.

The model is based on the well-known discrete symmetry group $A_{4}$ possessing the irreducible representations

$$
\begin{array}{lll}
\mathbf{1}: & S \rightarrow 1, & T \rightarrow 1, \\
\mathbf{1}^{\prime}: & S \rightarrow 1, & T \rightarrow \omega, \\
\mathbf{1}^{\prime \prime}: & S \rightarrow 1, & T \rightarrow \omega^{2}, \\
\mathbf{3}: & S \rightarrow \tilde{S}, & T \rightarrow \tilde{T},
\end{array}
$$

where

$$
\tilde{S}=\left(\begin{array}{ccc}
1 & 0 & 0 \\
0 & -1 & 0 \\
0 & 0 & -1
\end{array}\right), \quad \tilde{T}=\left(\begin{array}{ccc}
0 & 1 & 0 \\
0 & 0 & 1 \\
1 & 0 & 0
\end{array}\right)
$$

and $\omega=\exp (i 2 \pi / 3)$. Assigning the fields to the representations as

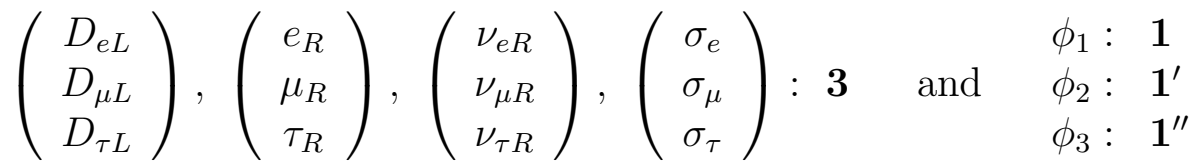

leads to the Yukawa Lagrangian

$$
\begin{aligned}
\mathcal{L}_{\text {Yukawa }}= & -y_{1}\left(\bar{D}_{e L} e_{R}+\bar{D}_{\mu L} \mu_{R}+\bar{D}_{\tau L} \tau_{R}\right) \phi_{1} \\
& -y_{2}\left(\bar{D}_{e L} e_{R}+\omega \bar{D}_{\mu L} \mu_{R}+\omega^{2} \bar{D}_{\tau L} \tau_{R}\right) \phi_{2} \\
& -y_{3}\left(\bar{D}_{e L} e_{R}+\omega^{2} \bar{D}_{\mu L} \mu_{R}+\omega \bar{D}_{\tau L} \tau_{R}\right) \phi_{3} \\
& -y_{4}\left(\bar{D}_{e L} \nu_{e R}+\bar{D}_{\mu L} \nu_{\mu R}+\bar{D}_{\tau L} \nu_{\tau R}\right) \tilde{\phi}_{1} \\
& -y_{5}\left(\bar{D}_{e L} \nu_{e R}+\omega^{2} \bar{D}_{\mu L} \nu_{\mu R}+\omega \bar{D}_{\tau L} \nu_{\tau R}\right) \tilde{\phi}_{2} \\
& -y_{6}\left(\bar{D}_{e L} \nu_{e R}+\omega \bar{D}_{\mu L} \nu_{\mu R}+\omega^{2} \bar{D}_{\tau L} \nu_{\tau R}\right) \tilde{\phi}_{3} \\
& -y_{7}\left(\bar{\nu}_{e R} C \bar{\nu}_{\mu R}^{T} \sigma_{\tau}+\bar{\nu}_{\mu R} C \bar{\nu}_{\tau R}^{T} \sigma_{e}+\bar{\nu}_{\tau R} C \bar{\nu}_{e R}^{T} \sigma_{\mu}\right)+\text { H.c., }
\end{aligned}
$$

\footnotetext{
${ }^{4}$ The two conditions (5) represent a total of four constraints (two from the real parts and two from the imaginary parts) on the neutrino masses and on lepton mixing. However, because those conditions implicitly involve the Majorana phases of the neutrinos, which are unobservable in oscillation experiments, the predictive power of our model is less than these four constraints might suggest.

${ }^{5}$ The boldface number inside each parentheses is the dimension of the gauge- $S U(2)$ representation; the second number is the weak hypercharge.
} 
where $C$ is the charge-conjugation matrix in Dirac space. The couplings $y_{1-7}$ are dimensionless. There are also bare Majorana mass terms

$$
\mathcal{L}_{\text {Majorana }}=-\frac{m}{2}\left(\bar{\nu}_{e R} C \bar{\nu}_{e R}^{T}+\bar{\nu}_{\mu R} C \bar{\nu}_{\mu R}^{T}+\bar{\nu}_{\tau R} C \bar{\nu}_{\tau R}^{T}\right)+\text { H.c. }
$$

where $m$ has mass dimension.

When the neutral components of $\phi_{1,2,3}$ get vacuum expectation values (VEVs) $v_{k}=\left\langle 0\left|\phi_{k}^{0}\right| 0\right\rangle$, the charged leptons acquire masses given by

$$
\begin{aligned}
& m_{e}=\left|y_{1} v_{1}+y_{2} v_{2}+y_{3} v_{3}\right| \\
& m_{\mu}=\left|y_{1} v_{1}+\omega y_{2} v_{2}+\omega^{2} y_{3} v_{3}\right| \\
& m_{\tau}=\left|y_{1} v_{1}+\omega^{2} y_{2} v_{2}+\omega y_{3} v_{3}\right|
\end{aligned}
$$

The three quantities $y_{k} v_{k}$ must be finetuned in order that $m_{e} \ll m_{\mu} \ll m_{\tau}$. 6

We assume the VEVs of the three $\sigma_{\alpha}$ to be equal (see appendix A): $\left\langle 0\left|\sigma_{e}\right| 0\right\rangle=\left\langle 0\left|\sigma_{\mu}\right| 0\right\rangle=\left\langle 0\left|\sigma_{\tau}\right| 0\right\rangle \equiv s$. We furthermore assume that $s$ is of the same order of magnitude as $m$, and that this order of magnitude is very large, viz. it is the seesaw scale. Thus, the subgroup $\mathbb{Z}_{3}$ of $A_{4}$ generated by $T$ is preserved at the high (seesaw) scale and only gets spontaneously broken at the low (Fermi 7 ) scale, when $\phi_{2}$ and $\phi_{3}$ acquire VEVs.

The neutrino mass matrices $M_{D}$ and $M_{R}$ are defined by

$$
\mathcal{L}_{\nu \text { mass }}=-\bar{\nu}_{R} M_{D} \nu_{L}-\frac{1}{2} \bar{\nu}_{R} M_{R} C \bar{\nu}_{R}^{T}+\text { H.c. }
$$

In our model,

$$
\begin{aligned}
M_{D} & =\operatorname{diag}(a, b, c), \\
M_{R} & =\left(\begin{array}{ccc}
m & m^{\prime} & m^{\prime} \\
m^{\prime} & m & m^{\prime} \\
m^{\prime} & m^{\prime} & m
\end{array}\right),
\end{aligned}
$$

\footnotetext{
${ }^{6}$ Most models require a finetuning in order to obtain $m_{e} \ll m_{\mu} \ll m_{\tau}$. Possible exceptions are models based on the Froggatt-Nielsen paradigm [6] and models with extra dimensions.

${ }^{7}$ Another possibility is that $v_{1,2,3}$ are much lower than the Fermi scale, if the quarks do not have Yukawa couplings to $\phi_{1,2,3}$ and only couple to an extra doublet $\phi_{0}$ which is invariant under $A_{4}$. In that scheme, the VEV of the neutral component of $\phi_{0}$ would be dominant in giving mass both to the gauge bosons and to the quarks, while the VEVs of the neutral components of $\phi_{1,2,3}$ would lie much below the Fermi scale. The masses of the components of the doublets $\phi_{1,2,3}$ would in this picture lie much above the Fermi scale, due to a type-II seesaw mechanism for Higgs doublets [7].
} 
where

$$
\begin{aligned}
a & =y_{4}^{*} v_{1}+y_{5}^{*} v_{2}+y_{6}^{*} v_{3}, \\
b & =y_{4}^{*} v_{1}+\omega y_{5}^{*} v_{2}+\omega^{2} y_{6}^{*} v_{3}, \\
c & =y_{4}^{*} v_{1}+\omega^{2} y_{5}^{*} v_{2}+\omega y_{6}^{*} v_{3}
\end{aligned}
$$

and $m^{\prime}=y_{7} s$. A seesaw mechanism takes place, whereupon an effective mass matrix for the light neutrinos

$$
M=-M_{D}^{T} M_{R}^{-1} M_{D}
$$

is generated. The matrix $A$ is then of the form ([6) , with

$$
t=\frac{m^{\prime 2}}{\left(m^{\prime}-m\right)\left(2 m^{\prime}+m\right)}
$$

The values of $a, b$, and $c$ are irrelevant for $A$.

One may, if one wants, furnish our model with an extra $C P$ symmetry,

$$
\begin{aligned}
& \left\{\begin{array} { l } 
{ D _ { e L } ( x ) \rightarrow \gamma _ { 0 } C \overline { D } _ { e L } ^ { T } ( \overline { x } ) , } \\
{ D _ { \mu L } ( x ) \rightarrow \gamma _ { 0 } C \overline { D } _ { \tau L } ^ { T } ( \overline { x } ) , } \\
{ D _ { \tau L } ( x ) \rightarrow \gamma _ { 0 } C \overline { D } _ { \mu L } ^ { T } ( \overline { x } ) , }
\end{array} \quad \left\{\begin{array}{l}
e_{R}(x) \rightarrow \gamma_{0} C \bar{e}_{R}^{T}(\bar{x}), \\
\mu_{R}(x) \rightarrow \gamma_{0} C \bar{\tau}_{R}^{T}(\bar{x}), \\
\tau_{R}(x) \rightarrow \gamma_{0} C \bar{\mu}_{R}^{T}(\bar{x}),
\end{array}\right.\right. \\
& \left\{\begin{array} { l } 
{ \nu _ { e R } ( x ) \rightarrow \gamma _ { 0 } C \overline { \nu } _ { e R } ^ { T } ( \overline { x } ) , } \\
{ \nu _ { \mu R } ( x ) \rightarrow \gamma _ { 0 } C \overline { \nu } _ { \tau R } ^ { T } ( \overline { x } ) , } \\
{ \nu _ { \tau R } ( x ) \rightarrow \gamma _ { 0 } C \overline { \nu } _ { \mu R } ^ { T } ( \overline { x } ) , }
\end{array} \quad \left\{\begin{array}{c}
\sigma_{e}(x) \rightarrow \sigma_{e}(\bar{x}), \\
\sigma_{\mu}(x) \rightarrow \sigma_{\tau}(\bar{x}), \\
\sigma_{\tau}(x) \rightarrow \sigma_{\mu}(\bar{x}), \\
\phi_{k}(x) \rightarrow \phi_{k}^{*}(\bar{x}) \forall k,
\end{array}\right.\right.
\end{aligned}
$$

where $x=(t, \vec{r})$ and $\bar{x}=(t,-\vec{r})$. This $C P$ symmetry renders $y_{1}, y_{2}, \ldots, y_{7}$, and $m$ real. The mass $m^{\prime}$ will then be real, because the scalars $\sigma_{\alpha}$ are Hermitian fields, hence their VEV $s$ is real. Even if the $C P$ symmetry is spontaneously broken by (relatively) complex $v_{1}, v_{2}$, and $v_{3}$, the ensuing phases of $a, b$, and $c$ may be withdrawn from $M$ through appropriate rephasings of the light-neutrino fields. Thus, there exists a restriction of our model in which $M$ is real.

We proceed to fit the predictions of our model, viz. eqs. (5), to the phenomenological data. The neutrino masses are $m_{1,2,3}$. We use the standard parameterization of lepton mixing in ref. [8], through three mixing angles $\theta_{12}, \theta_{13}$, and $\theta_{23}$ and one $C P$-violating phase $\delta$. We have used the following 
allowed ranges for the various observables:

$$
\begin{aligned}
6.99 \times 10^{-5} \mathrm{eV}^{2} & \leq m_{2}^{2}-m_{1}^{2} \leq 8.20 \times 10^{-5} \mathrm{eV}^{2} \\
2.16 \times 10^{-3} \mathrm{eV}^{2} & \leq\left|m_{3}^{2}-m_{1}^{2}\right| \leq 2.74 \times 10^{-3} \mathrm{eV}^{2} \\
0.25 & \leq \sin ^{2} \theta_{12} \leq 0.37 \\
0.016 & \leq \sin ^{2} \theta_{13} \leq 0.033 \\
0.33 & \leq \sin ^{2} \theta_{23} \leq 0.68
\end{aligned}
$$

These ranges simultaneously encompass all the corresponding $3 \sigma$ ranges furnished by the relevant phenomenological analyses [9, 10, 11]. We stress that, even though the fit presented here uses these quite ample ranges, we have also found that most observables easily fall within their respective $1 \sigma$ ranges given in, for instance, ref. [9]; the exception is the mixing angle $\theta_{12}$, which is in our model rather large. We have left $\delta$ free, even though refs. [9, 11] provide some bounds on it, which are, however, valid only at the $1 \sigma$ level.

Our first finding is that in our model the phase $\delta$ must be close to either 0 or $\pi: 8$ if $\delta \approx 0$ then $\theta_{23}$ is in the first octant while $\theta_{23}$ is in the second octant when $\delta \approx \pi 9$ This can be seen in the scatter plot of fig. 1. Therefrom one gathers that in our model $|\cos \delta| \gtrsim 0.7$ (0.8 if the neutrino mass spectrum is inverted) and $\left|\sin ^{2} \theta_{23}-0.5\right| \gtrsim 0.08$.

In fig. 2 one sees that our model is unable to predict $\theta_{13}$ but neatly predicts $\sin ^{2} \theta_{12} \gtrsim 0.34$. This is a rather large value, which is allowed by the phenomenological data only at the $2 \sigma$ (or even $3 \sigma$ ) level. It can moreover be seen in fig. 2 (and also in fig. 4) that the restricted real version of our model does not have much more predictive power than the general version, even though it has one degree of freedom less.

In the right panel of fig. 2 one observes that the lower bound on $\theta_{12}$ in our model coincides with the prediction for $\theta_{12}$ in a model with trimaximal mixing $\left(\mathrm{TM}_{2}\right.$ in the nomenclature of ref. [12]). Trimaximal mixing is defined to be the situation where $\left|U_{\alpha 2}\right|=3^{-1 / 2} \forall \alpha \in\{e, \mu, \tau\}$. A model with $\mathrm{TM}_{2}$ has been suggested a few years ago [13]. In $\mathrm{TM}_{2}\left|U_{e 2}\right|^{2}=\sin ^{2} \theta_{12} \cos ^{2} \theta_{13}=$ $1 / 3$ and therefore $\sin ^{2} \theta_{12} \approx 0.34$, which is a bit large but has not deterred several authors - see for instance ref. [14] - from having recently suggested models and Ansätze featuring $\mathrm{TM}_{2}$. Note that $\mathrm{TM}_{2}$, just as our model, is

\footnotetext{
${ }^{8}$ The predictions of our model are symmetric under $\mu \leftrightarrow \tau$. In the parameterization of the lepton mixing matrix that we use, the $\mu \leftrightarrow \tau$ interchange corresponds to $\cos \delta \rightarrow-\cos \delta$ and $\sin ^{2} \theta_{23} \rightarrow 0.5-\sin ^{2} \theta_{23}$. This symmetry is easily observable in figs. 1 and 3 and in the left panel of fig. 4

${ }^{9}$ This contradicts the phenomenological findings (at the $1 \sigma$ level) of ref. [9, according to which $\theta_{23}$ lies in the first octant and $\delta$ is close to $\pi$. However, those findings are not in agreement with the ones of refs. [10, 11].
} 


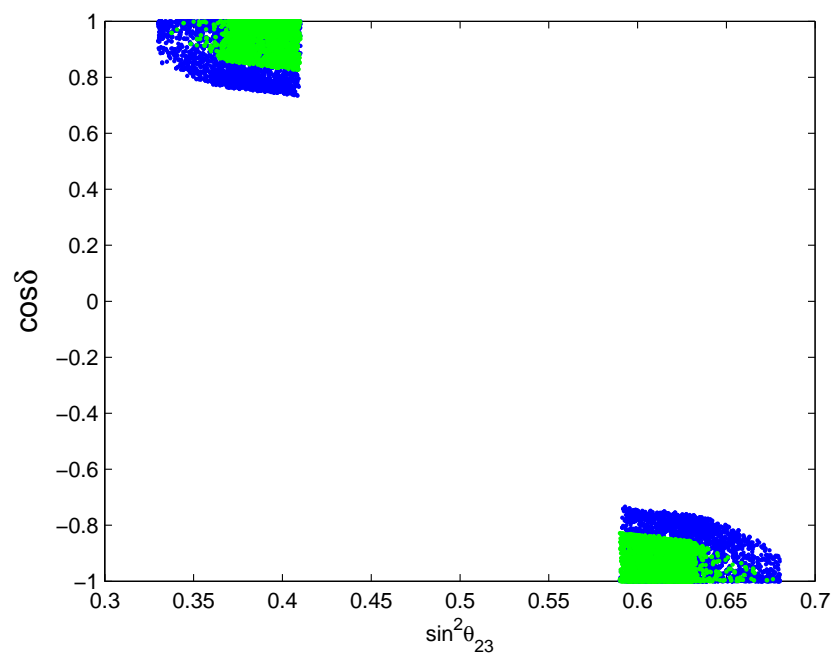

Figure 1: Scatter plot of $\cos \delta$ against $\sin ^{2} \theta_{23}$. The green (light grey) points are for an inverted neutrino mass spectrum $\left(m_{3}<m_{1,2}\right)$, the blue (black) points for a normal one $\left(m_{3}>m_{1,2}\right)$; this convention is used in all the figures of this paper.

characterized by predictions for lepton mixing which are invariant under any permutation of the lepton flavours.

One further prediction of $\mathrm{TM}_{2}$ is 10

$$
(\cos \delta) \tan \left(2 \theta_{23}\right)=\frac{\cos \left(2 \theta_{13}\right)}{\left(\sin \theta_{13}\right) \sqrt{3 \cos ^{2} \theta_{13}-1}}>0 .
$$

Therefore, in $\mathrm{TM}_{2}$, just as in our model, $\delta$ is in the first (or fourth) quadrant when $\theta_{23}$ is in the first octant, and $\delta$ is in the second (or third) quadrant when $\theta_{23}$ is in the second octant. Moreover, in $\mathrm{TM}_{2} \theta_{23}$ becomes closer to $\pi / 4$ when $|\cos \delta|$ becomes smaller.

Figure 3 shows that, if we want a lower $\theta_{12}$ in our model, then we must accept $|\cos \delta|$ to be ever closer to 1, i.e. a more stringent phenomenological upper bound on $\sin ^{2} \theta_{12}$ translates in our model into a more stringent lower bound on $|\cos \delta|$.

In fig. 4 one sees that our model's bound on $\theta_{23}$ depends only faintly on $\theta_{13}$. One also sees that our model's lower bound on $\left|0.5-\sin ^{2} \theta_{23}\right|$ coincides with the prediction of $\mathrm{TM}_{2}$ for $\left|0.5-\sin ^{2} \theta_{23}\right|$ when $|\cos \delta|$ is maximal.

Figure 5 shows the scale of the neutrino masses in our model.

\footnotetext{
${ }^{10}$ Reference [15] contains relations that generalize eq. (21).
} 

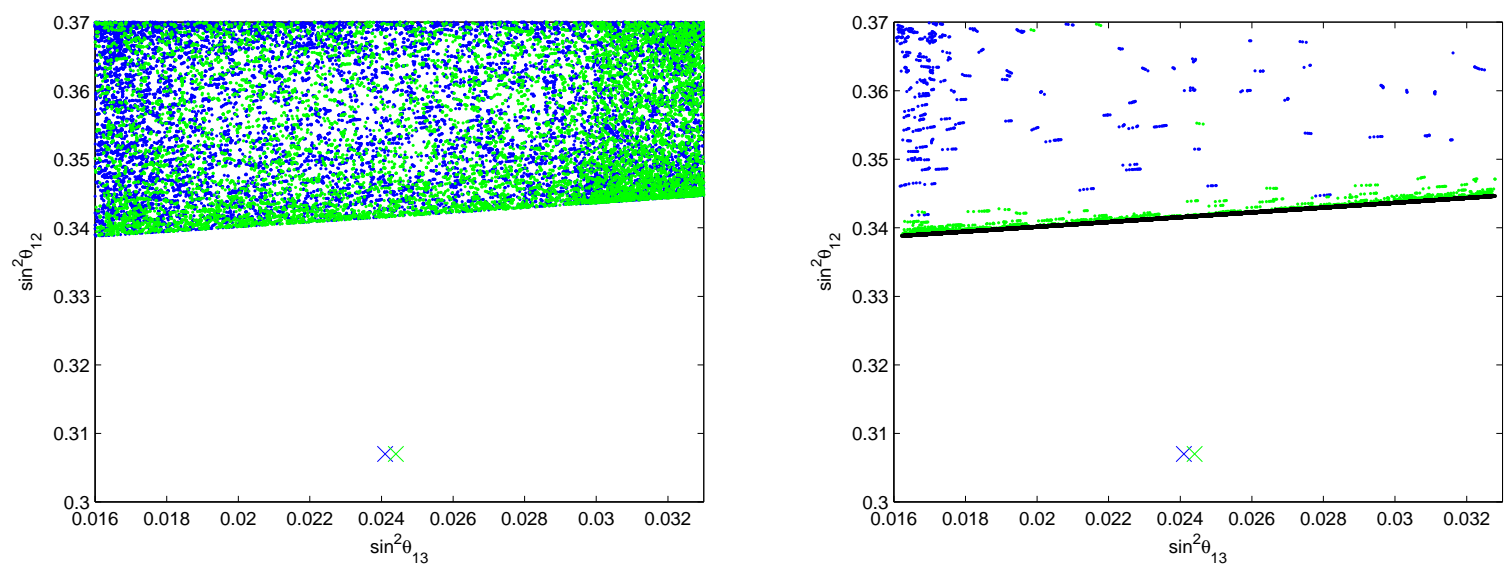

Figure 2: Scatter plots of $\sin ^{2} \theta_{12}$ against $\sin ^{2} \theta_{13}$. The left plot is for the general (complex) version of the model, the right plot is for the real version with $\cos \delta=-1$. The black line in the right plot displays the prediction [13] $\sin ^{2} \theta_{12}\left(1-\sin ^{2} \theta_{13}\right)=1 / 3$ of trimaximal mixing $\left(\mathrm{TM}_{2}\right)$. The crosses (blue (black) for a normal neutrino mass spectrum, green (light grey) for an inverted spectrum) indicate the phenomenological best-fit points of ref. [9].

We next speculate on possible experimental indications for (or against) our model. As is clear in the first three lines of eq. (10), in our model the Yukawa couplings of the neutral scalars to the charged leptons conserve flavour. Therefore, flavour-changing neutral Yukawa interactions only arise at loop level and are suppressed by a loop factor $\left(16 \pi^{2}\right)^{-1}$ and by two additional Yukawa couplings. One may, moreover, show [16] that the (loop induced) flavour changing neutral couplings of the charged leptons to the photon and to the $Z^{0}$ are suppressed by inverse powers of the seesaw scale and are therefore, in general, unobservably small. Therefore, no decays like $\mu \rightarrow e \gamma$ or $Z^{0} \rightarrow$ $e^{+} \mu^{-}$are expected, but decays like $h \rightarrow \tau^{+} \mu^{-}$might be observable at the LHC ( $h$ is the observed scalar particle with mass $125 \mathrm{GeV}$ ).

When extending our model to the quark sector, one may either add to it further Higgs doublets or - a more economic possibility - make the quarks have Yukawa couplings to any one of the Higgs doublets $\phi_{1}, \phi_{2}$, or $\phi_{3}$ (or else the up-type quarks may couple to one of them and the down-type quarks to another one). The fact that our Higgs doublets are singlets of the flavour group allows for this inviting possibility. Depending on the specific Yukawa couplings used, the signatures of the model at the LHC will vary. It is worth pointing out that, in any multi-Higgs-doublet model (MHDM), a physical scalar couples to $Z^{0} Z^{0}$ with a coupling at most as strong as the one of 


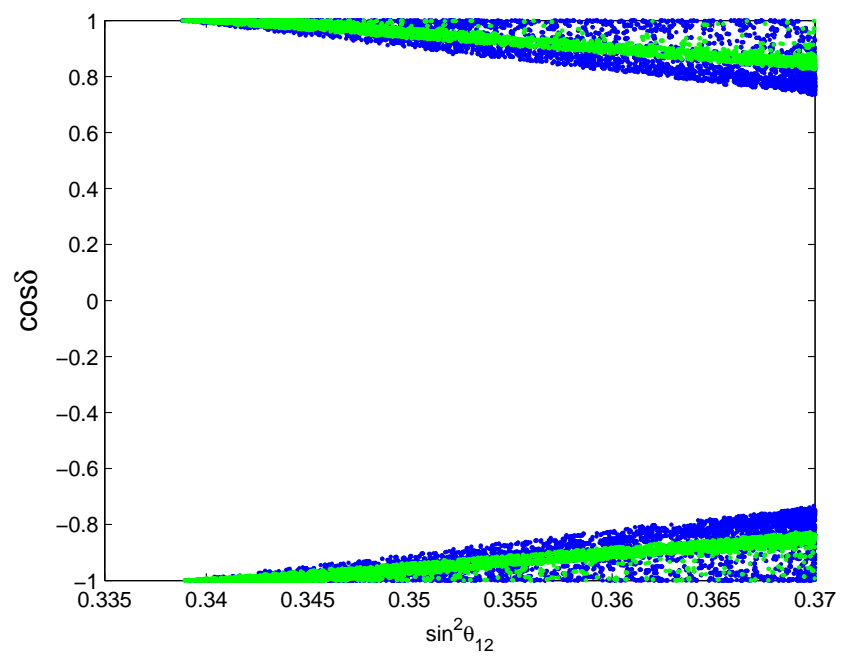

Figure 3: Scatter plot of $\cos \delta$ against $\sin ^{2} \theta_{12}$.
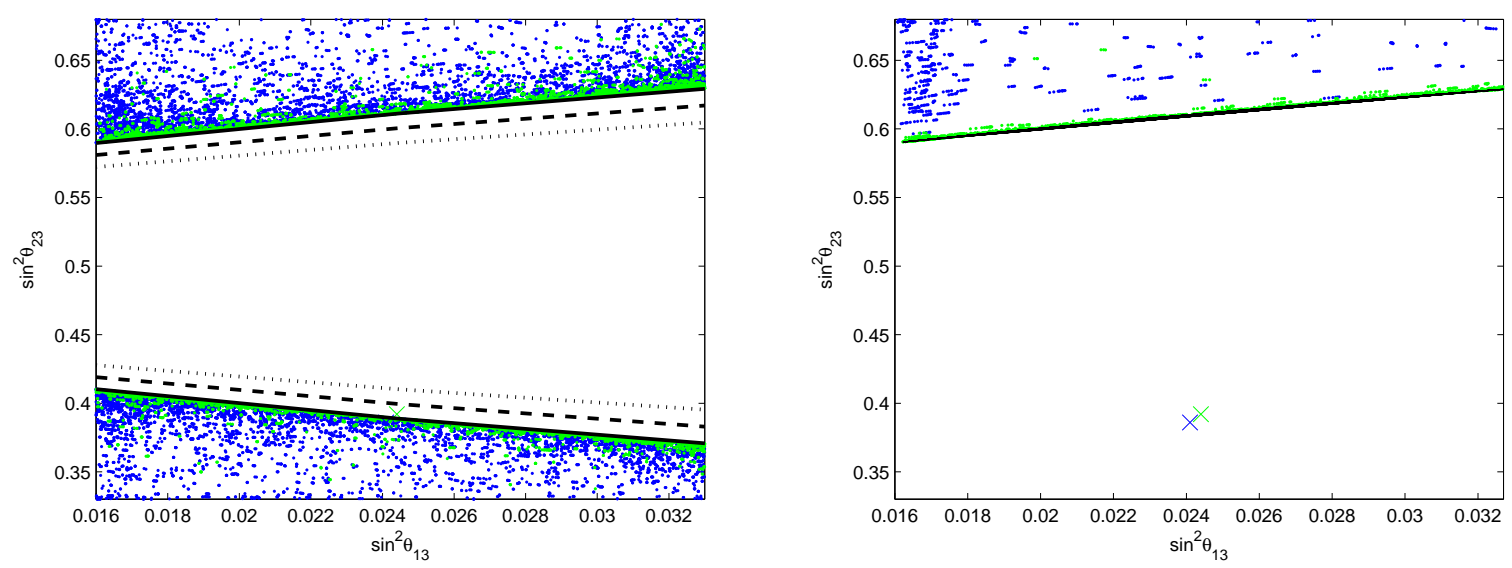

Figure 4: Scatter plots of $\sin ^{2} \theta_{23}$ against $\sin ^{2} \theta_{13}$. The left plot is for the general version and the right plot is for the real one with $\cos \delta=-1$. The black lines correspond to eq. (21), predicted by $\mathrm{TM}_{2}$ [13], when $\cos \delta= \pm 1$ (full lines), $\cos \delta= \pm 0.9$ (dashed lines), and $\cos \delta= \pm 0.8$ (dotted lines). The crosses indicate the phenomenological best-fit points of ref. [9]. 

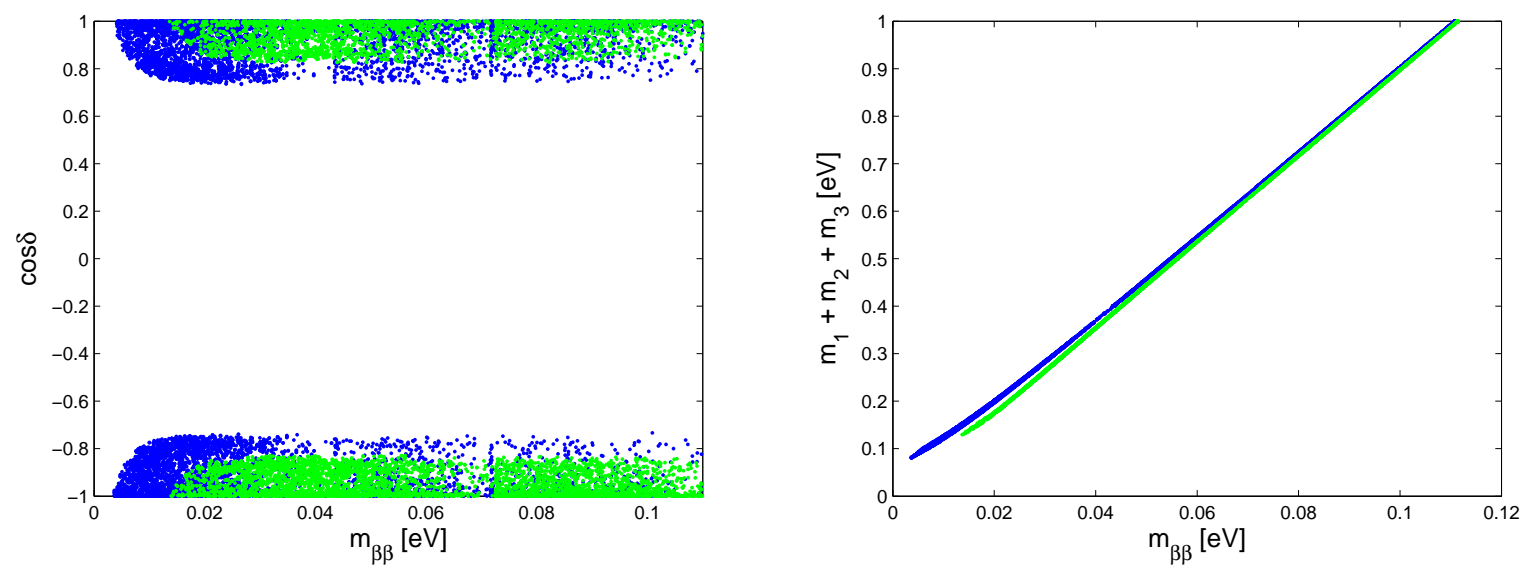

Figure 5: Ranges of allowed masses of the neutrinos in our model. The left scatter plot shows the neutrino mass relevant for neutrinoless double beta decay as a function of $\cos \delta$; the right plot correlates that quantity with the sum of the three light neutrino masses. We have imposed the cut $m_{1}+m_{2}+m_{3} \leq 1 \mathrm{eV}$ on the sum of the neutrino masses.

the Higgs particle of the Standard Model (SM) [17]. This naively suggests that the observed decay $h \rightarrow Z^{0} Z^{0}$ could be used to strongly constrain the parameter space of a MHDM, but this is not the case. Indeed, in a MHDM the couplings of any particular scalar to the top and bottom quarks might be either (much) stronger or (much) weaker than in the SM, and therefore in a MHDM both the production cross section and the total decay width of $h$ will in general be at variance with those expected in the SM.

To summarize, we have presented in this paper a seesaw model featuring a simple application of the $A_{4}$ symmetry and which makes the predictions (5) for lepton mixing. We have shown that our model is compatible with experiment provided $\theta_{12}$ is in the upper part of its allowed range. Our model predicts non-maximal $\theta_{23}$ and also makes the prediction that $|\cos \delta|$ is very close to 1.

\section{Acknowledgements:}

LL thanks João P. Silva for a useful discussion. The work of PMF is supported by the Portuguese Foundation for Science and Technology (FCT) through the projects PEst-OE/FIS/UI0618/2011, PTDC/FIS/117951/2010, and the FP7 Reintegration Grant PERG08-GA-2010-277025. The work of LL 
is supported through the Marie Curie Initial Training Network "UNILHC" PITN-GA-2009-237920 and through the projects PEst-OE/FIS/UI0777/2013, PTDC/FIS-NUC/0548/2012, and CERN/FP/123580/2011 of FCT. The work of POL is supported by the Austrian Science Fund (FWF) through the project P 24161-N16.

\section{A Appendix: the VEVs of the $\sigma_{\alpha}$}

The potential for the $\sigma_{\alpha}$ is

$$
\begin{aligned}
V_{\sigma}= & \mu\left(\sigma_{e}^{2}+\sigma_{\mu}^{2}+\sigma_{\tau}^{2}\right)+\lambda_{1}\left(\sigma_{e}^{2}+\sigma_{\mu}^{2}+\sigma_{\tau}^{2}\right)^{2} \\
& +\tilde{m} \sigma_{e} \sigma_{\mu} \sigma_{\tau}+\lambda_{2}\left(\sigma_{e}^{2} \sigma_{\mu}^{2}+\sigma_{\mu}^{2} \sigma_{\tau}^{2}+\sigma_{\tau}^{2} \sigma_{e}^{2}\right) .
\end{aligned}
$$

We have neglected terms which include both the $\sigma_{\alpha}$ and the $\phi_{k}$ since the VEVs of the $\phi_{k}$ should be much smaller than the VEVs of the $\sigma_{\alpha}$ and therefore those terms should have a negligible influence on the equations which determine the VEVs of the $\sigma_{\alpha}$.

One may parameterize the VEVs of the $\sigma_{\alpha}$ as

$$
\left\langle 0\left|\sigma_{e}\right| 0\right\rangle=U \cos \vartheta, \quad\left\langle 0\left|\sigma_{\mu}\right| 0\right\rangle=U \sin \vartheta \cos \varphi, \quad\left\langle 0\left|\sigma_{\tau}\right| 0\right\rangle=U \sin \vartheta \sin \varphi,
$$

with $U \geq 0, \vartheta \in[0, \pi]$, and $\varphi \in[0,2 \pi[$. Then,

$$
\begin{aligned}
V_{\sigma 0} \equiv\left\langle 0\left|V_{\sigma}\right| 0\right\rangle= & \mu U^{2}+\lambda_{1} U^{4}+\frac{\tilde{m} U^{3}}{2} \sin ^{2} \vartheta \cos \vartheta \sin 2 \varphi \\
& +\lambda_{2} U^{4} \sin ^{2} \vartheta\left(\cos ^{2} \vartheta+\frac{1}{4} \sin ^{2} \vartheta \sin ^{2} 2 \varphi\right) .
\end{aligned}
$$

There is a range of $\tilde{m}$ and $\lambda_{2}$ for which the minimum of $V_{\sigma 0}$ occurs when $\sin 2 \varphi$ is at the boundary of its range, viz. when $\sin 2 \varphi=1$. There,

$$
V_{\sigma 0}=\mu U^{2}+\lambda_{1} U^{4}+\frac{\tilde{m} U^{3}}{2} \sin ^{2} \vartheta \cos \vartheta+\frac{\lambda_{2} U^{4}}{4} \sin ^{2} \vartheta\left(1+3 \cos ^{2} \vartheta\right)
$$

Then,

$$
\frac{\partial V_{\sigma 0}}{\partial \cos \vartheta}=U^{3}\left(1-3 \cos ^{2} \vartheta\right)\left(\frac{\tilde{m}}{2}+\lambda_{2} U \cos \vartheta\right) .
$$

Within a range of $\tilde{m}$ and $\lambda_{2}$ the minimum of $V_{\sigma 0}$ occurs when $\cos ^{2} \vartheta=1 / 3$ and, indeed, $\cos \vartheta=1 / \sqrt{3}$. In this way one obtains $\left\langle 0\left|\sigma_{e}\right| 0\right\rangle=\left\langle 0\left|\sigma_{\mu}\right| 0\right\rangle=$ $\left\langle 0\left|\sigma_{\tau}\right| 0\right\rangle=U / \sqrt{3}$ as desired. 


\section{References}

[1] Y. Abe et al. (Double Chooz Coll.), Indication for the disappearance of reactor electron antineutrinos in the Double Chooz experiment, Phys. Rev. Lett. 108 (2012) 131801;

F.P. An et al. (Daya Bay Coll.), Observation of electron-antineutrino disappearance at Daya Bay, Phys. Rev. Lett. 108 (2012) 171803;

J.K. Ahn et al. (RENO Coll.), Observation of reactor electron antineutrino disappearance in the RENO experiment, Phys. Rev. Lett. 108 (2012) 191802.

[2] F. Feruglio, C. Hagedorn, Y. Lin, and L. Merlo, Lepton flavour violation in a supersymmetric model with $A_{4}$ flavour symmetry, Nucl. Phys. B 832 (2010) 251;

S.F. King and C. Luhn, $A_{4}$ models of tri-bimaximal-reactor mixing, J. High Energy Phys. 1203 (2012) 036;

M.-C. Chen, J. Huang, J.-M. O'Bryan, A.M. Wijangco, and F. Yu, Compatibility of $\theta_{13}$ and the type I seesaw model with $A_{4}$ symmetry, J. High Energy Phys. 1302 (2013) 021;

M. Holthausen, M. Lindner, and M.A. Schmidt, Lepton flavor at the electroweak scale: A complete $A_{4}$ model, Phys. Rev. D 87 (2013) 033006; S.F. King, S. Morisi, E. Peinado, and J.W.F. Valle, Quark-lepton mass relation in a realistic $A_{4}$ extension of the Standard Model, Phys. Lett. B 724 (2013) 68;

S. Morisi, M. Nebot, K.M. Patel, E. Peinado, and J.W.F. Valle, Quarklepton mass relation and CKM mixing in an $A_{4}$ extension of the Minimal Supersymmetric Standard Model, Phys. Rev. D 88, 036001 (2013).

[3] D. Meloni, S. Morisi, and E. Peinado, Neutrino phenomenology and stable dark matter with $A_{4}$, Phys. Lett. B 697 (2011) 339;

S. Gupta, A.S. Joshipura and K.M. Patel, Minimal extension of tribimaximal mixing and generalized $Z_{2} \times Z_{2}$ symmetries, Phys. Rev. D 85 (2012) 031903;

H. Ishimori and E. Ma, New simple $A_{4}$ neutrino model for nonzero $\theta_{13}$ and large $\delta_{C P}$, Phys. Rev. D 86 (2012) 045030;

E. Ma, Self-organizing neutrino mixing matrix, Phys. Rev. D 86 (2012) 117301;

E. Ma, A. Natale, and A. Rashed, Scotogenic $A_{4}$ neutrino model for nonzero $\theta_{13}$ and large $\delta_{C P}$, Int. J. Mod. Phys. A 27 (2012) 1250134;

Y. Ben Tov, X.-G. He, and A. Zee, An $A_{4} \times \mathbb{Z}_{4}$ model for neutrino mixing, J. High Energy Phys. 1212 (2012) 093;

Y.H. Ahn, S.K. Kang, and C.S. Kim, Spontaneous $C P$ violation in $A_{4}$ 
flavor symmetry and leptogenesis, Phys. Rev. D 87 (2013) 113012;

A.E. Carcamo Hernandez, I.d.M. Varzielas, S.G. Kovalenko, H. Päs and I. Schmidt, Lepton masses and mixings in a $A_{4}$ multi-Higgs model with radiative seesaw, arXiv:1307.6499 [hep-ph].

[4] K.S. Babu, E. Ma, and J.W.F. Valle, Underlying $A_{4}$ symmetry for the neutrino mass matrix and the quark mixing matrix, Phys. Lett. B 552 (2003) 207;

For a modern version, see S. Morisi, D.V. Forero, J.C. Romão, and J.W.F. Valle, Neutrino mixing with revamped $A_{4}$ flavour symmetry, Phys. Rev. D 88 (2013) 016003.

[5] P.M. Ferreira, L. Lavoura, and P.O. Ludl, Five models for lepton mixing, J. High Energy Phys. 1308 (2013) 113.

[6] C.D. Froggatt and H.B. Nielsen, Hierarchy of quark masses, Cabibbo angles and CP violation, Nucl. Phys. B 147 (1979) 277.

[7] W. Grimus, L. Lavoura, and B. Radovčić, Type II seesaw mechanism for Higgs doublets and the scale of new physics, Phys. Lett. B 674 (2009) 117.

[8] J. Beringer et al. (Particle Data Group), Review of particle physics, Phys. Rev. D 86 (2012) 010001.

[9] G.L. Fogli, E. Lisi, A. Marrone, D. Montanino, A. Palazzo, and A.M. Rotunno, Global analysis of neutrino masses, mixings and phases: Entering the era of leptonic CP violation searches, Phys. Rev. D 86 (2012) 013012 .

[10] D.V. Forero, M. Tórtola, and J.W.F. Valle, Global status of neutrino oscillation parameters after Neutrino-2012, Phys. Rev. D 86 (2012) 073012 .

[11] M.C. Gonzalez-Garcia, M. Maltoni, J. Salvado, and T. Schwetz, Global fit to three neutrino mixing: Critical look at present precision, J. High Energy Phys. 1212 (2012) 123.

[12] C.H. Albright, A. Dueck, and W. Rodejohann, Possible alternatives to tri-bimaximal mixing, Eur. Phys. J. C 70 (2010) 1099.

[13] W. Grimus and L. Lavoura, A model for trimaximal lepton mixing, J. High Energy Phys. 0809 (2008) 106. 
[14] N. Memenga, W. Rodejohann, and H. Zhang, $A_{4}$ flavor symmetry model for Dirac neutrinos and sizable $U_{e 3}$, Phys. Rev. D 87 (2013) 053021; S.F. King, T. Neder, and A.J. Stuart, Lepton mixing predictions from $\Delta\left(6 n^{2}\right)$ family symmetry, arXiv:1305.3200 [hep-ph];

H. Qu and B.-Q. Ma, New mixing pattern for neutrinos, Phys. Rev. D 88 (2013) 037301.

[15] S.-F. Ge, D.A. Dicus, and W.W. Repko, $\mathbb{Z}_{2}$ symmetry prediction for the leptonic Dirac CP phase, Phys. Lett. B 702 (2011) 220;

S.-F. Ge, D.A. Dicus, and W.W. Repko, Residual symmetries for neutrino mixing with a large $\theta_{13}$ and nearly maximal $\delta_{D}$, Phys. Rev. Lett. 108 (2012) 041801.

[16] W. Grimus and L. Lavoura, Soft lepton flavor violation in a multi-Higgsdoublet seesaw model, Phys. Rev. D 66 (2002) 014016;

R. Alonso, M. Dhen, M.B. Gavela, and T. Hambye, Muon conversion to electron in nuclei in type-I seesaw models, J. High Energy Phys. 1301 (2013) 118.

[17] See for instance W. Grimus, L. Lavoura, O.M. Ogreid, and P. Osland, A precision constraint on multi-Higgs-doublet models, J. Phys. G 35 (2008) 075001;

G.C. Branco, P.M. Ferreira, L. Lavoura, M.N. Rebelo, M. Sher, and J.P. Silva, Theory and phenomenology of two-Higgs-doublet models, Phys. Rep. 516 (2012) 1. 